\title{
Eye gaze and viewpoint in multimodal interaction management
}

\begin{abstract}
In this paper, we present a strongly embodied take on the phenomenon of viewpoint by exploring the role of eye gaze in face-to-face conversation, in relation to and interaction with other expressive modalities. More specifically, we look into specific gaze patterns, as well as gaze synchronization with speech, as instruments in the negotiation of speakership and participant roles in interaction. In order to get fine-grained information on the different modalities under scrutiny, we used the InSight Interaction Corpus (authors 2015). This multimodal video corpus consists of two- and three-party interactions (in Dutch), with head-mounted scene cameras and eye-trackers tracking all participants' visual behavior, providing a unique 'speaker-internal' perspective on the conversation.

The analysis of interactional sequences from the corpus (dyads and triads) reveals specific patterns of gaze distribution related to the temporal organization of viewpoint in dialogue. Different dialogue acts typically display specific gaze events at crucial points in time, as e.g. in the case of brief gaze aversion associated with turn-holding, and shared gaze between interlocutors at the critical point of turn-taking. In addition, the data show a strong correlation and temporal synchronization between eye gaze and speech in the realization of specific dialogue acts, as shown by means of a series of cross-recurrence analyses for specific turn-holding mechanisms (e.g. verbal fillers co-occurring with brief moments of gaze aversion).
\end{abstract}

\section{Introduction}

The different research fields involved in the study of human communication, including linguistics, sociology, psychology, communication studies, and human-computer interaction research, among others, have all pointed at the intrinsic interactional and multimodal nature of natural communication. The interactional aspect pertains to the coordination that is required to arrive at successful communication, grounded in joint actions (Clark 1996; Pickering \& Garrod 2004; Linell 2009; Verhagen 2005, 2008; see Authors 2015, Authors 2016 for overviews of how Cognitive Linguistics has dealt with interactional language use). Communication partners coordinate their production and interpretation, that is, they need to be able to react or adjust to their partners as much as they need to be able to anticipate their partners' actions. Establishing and managing this interaction typically requires information from different signal systems simultaneously, such as speech and other auditory signals, gesture, manual signs, body posture, etc. Accounting for the interplay between these different modes of representation in a multimodal approach is gradually drawing scholars' attention in various fields, including conversation analysis (e.g. Deppermann 2013a) and Cognitive Linguistics (Pinar Sanz 2013, Dancygier \& Sweetser 2015).

The focus of multimodal studies on spoken interactional language has mainly been on the form and function of gestures, and more specifically hand gestures, and how these gestures relate to the speech they typically accompany (co-speech gestures) (Kendon 2004, McNeill 2005, Cienki \& Müller 2008, Müller 
et al. 2014, a.o.). Other relevant nonverbal means of representation have not received the same systematic scrutiny, even if they have long been recognized as interactionally relevant articulators. This is also the case for the role of eye gaze in interaction, which has been attributed an important role as a coordinating device in different interactional settings, such as face-to-face spoken and signed conversations (Rossano 2012a, Goodwin 1980, Baker 1977, Emmorey et al. 2009), human-computer interaction (Bailly et al. 2010, Vertegaal et al. 2001) and musical interactions (Kawase 2014a/b, King \& Ginsborg 2011). In each of these interaction types, gaze has been shown to serve a variety of functions, including the negotiation of speakership (or leadership in musical interaction), feedback and feedback elicitation, signaling attention and interest, etc. A major challenge, however, resides in gaining access to the complex and continuous stream of eye gaze data that are simultaneously produced by the different participants in the interaction. Providing a detailed analysis of gaze patterns, both in relation to cooccurring speech and gesture produced by the same subject (resulting in recurrent multimodal cooccurrences) and in relation to co-participant behavior (resulting in multimodal interactional correlations), may provide relevant new insights for any kind of usage-based language study interested in how different viewpoints are synchronized in interaction.

In this paper, we develop a multimodal account of the role of eye gaze in face-to-face conversations, with a specific focus on how this semiotic channel (to use Langacker's 2001 term) relates to and interacts with other expressive modalities. More specifically, we zoom in on measurable gaze events, such as brief moments of gaze aversion, and how these are temporally synchronized with verbal markers that are known to serve specific interactional functions (e.g. fillers such as euhm, which have been described as nonlinguistic signals to hold, take or yield the turn in conversation, Clark \& Fox Tree 2002, Schegloff 1982, Beattie 1983). The aim of this specific case study is to gain insights into the way in which communicative functions may be realized by different means simultaneously, realizing multimodal packages (McNeill 2006). In other words, we want to provide empirical evidence for Clark's claim on composite signals in natural language production:

Most signals are composite signals, the artful fusion of two or more methods of signaling. [...] Some might conclude that the nonlinguistic methods are crude, unsystematic, ad hoc, and marginal, and deserve to be relegated to the periphery of language use. This wouldn't be right. On the contrary, the nonlinguistic methods are subtle, highly systematic, and not at all ad hoc. And they are part and parcel of most signals that are usually classified as "linguistic" (Clark 1996: 156).

In order to get fine-grained information on the different modalities under scrutiny, we will use a specific type of data set that provides detailed access to interactants' gaze behavior through the use of mobile eye-tracking data. The InSight Interaction Corpus (authors 2015, see section 3 ) is a multimodal video corpus consisting of two- and three-party interactions (in Dutch), with head-mounted scene cameras and eye-trackers tracking all participants' visual behavior, providing a unique 'speaker-internal' perspective on the conversation. A selection of sequences from this corpus is used to explore the correlation and 
temporal synchronization between measurable gaze events and specific turn management strategies. The latter will be approached both from the perspective of conversational units realizing specific interactional functions (i.e. intonation units with a turn-holding or turn-giving function) and by focusing on specific verbal elements that may serve such an interactional function.

The remainder of this paper is structured as follows. In section 2, we discuss relevant work on verbal and multimodal strategies for interaction management. Section 3 presents the research aim and methodology for this study, including the multimodal corpus data that were used. Section 4 consists of two empirical subsections, one presenting a general distributional analysis for gaze patterns and different types of dialogue acts (4.1.) and one reporting on a time-sensitive study that explored the temporal relationship between relevant gaze events and turn management strategies (4.2.). The paper closes off with a section that discusses the implications and broader potential of this type of cognitively inspired multimodal interaction analysis.

\section{Multimodal interaction management}

Since the 1960s, researchers in the fields of conversation analysis, ethnomethodology and interactional linguistics have been studying the dynamics of naturally occurring human interaction, including the strategies that are employed to manage such exchanges (see Drew \& Heritage 2006, Sidnell \& Stivers 2013 for recent overviews). Interaction management or turn management is used here in the broad sense to refer to the coordinated efforts of participants to assure a fluent, synchronized interaction that leads to successful communication. One of the key features of this coordinated action is its sequential organization, as described in early conversation analysis (Sacks et al. 1974). Sequence organization essentially involves a form of turn-taking between the participants in an interaction, ideally resulting in alternating turns with minimal overlap between the speakers. Over the last decades, conversation analysis has described, in great detail, the practices that interlocutors use in constructing and allocating (i.e. selecting next speakership) turns in interaction. These include considerations on the units in interaction (e.g. intonation units, Turn-Constructional Units (TCUs), turns-at-talk), the identification of transitionrelevance places (i.e. points where a turn may end, and thus a new speaker might take over) and the strategies for taking, giving or holding a turn in conversation.

The vast majority of studies on interaction management deal exclusively with the verbal level of analysis, basing the inductive analysis on detailed transcriptions of audio- or video-recorded slices of natural conversation. Somewhat symptomatic, in this sense, is the definition of turn-taking in the classical paper by Sacks, Schegloff \& Jefferson, which refers to human interaction in terms of 'speech exchange systems', thus relegating the nonverbal aspects of exchange to the periphery of investigation.

Turn-taking is used for the ordering of moves in games, for allocating political office, for regulating traffic at intersections, for serving customers at business establishments, and for 
talking in interviews, meetings, debates, ceremonies, conversations etc. -these last being members of the set which we shall refer to as 'speech exchange systems'. (Sacks, Schegloff \& Jefferson 1974: 696)

Although most of the work in conversation analysis focused on the (para)verbal signals guiding the 'speech exchange system', there was an early awareness of the intrinsic multimodal nature of interaction management. ${ }^{1}$ For instance, Jefferson (1972, cited in Schmitt 2005:22), in her analysis of summonsanswer sequences, argues that "[t]here are various items that are appropriately used as answers, e.g. "Yes?", "What", "Uh huh?", turning of the eyes or of the body to face the beckoner". Examples such as this show that verbal strategies are inextricably bound up with embodied practices in interaction. What is more, as convincingly argued by Schmitt (2005), extending the analytical scope to the full complexity of multimodal behavior may in fact challenge some of the key assumptions and call for modifications of the turn-taking machinery as described so far.

In a more recent development, conversation analysis and interactional linguistics have started to develop a growing interest in a systematic multimodal approach to turn-taking, based on the detailed analysis of video data. Topics that are addressed include the role of gesture and posture in the organization of turntaking (e.g. Mondada 2007 and 2013 on pointing as a resource for selection and self-selection) and turn construction (Deppermann 2013b), the synchronization of verbal and embodied resources both within and across participants involved in interaction (Streeck 2009, Schegloff 2007) and the collaborative realization of multimodal packages in interaction, i.e. the co-construction of dialogue units or idea units across semiotic resources (McNeill 2006, Quek et al. 2005). As providing a detailed review of the literature on these topics would extend the scope of this paper (see Mondada 2013 for a recent overview), the remainder of this section will zoom in on work that has addressed the specific role of eye gaze as a communicative resource in interaction, both on the part of speakers and their addressees.

Early pioneering work in conversation analysis by Kendon (1967), Argyle \& Cook (1976) and Goodwin $(1980,1981)$, based on transcriptions of video data, presented the first systematic data on the direction of speakers' and hearers' gaze during face-to-face conversation. These studies singled out a variety of functions of eye gaze as a communicative instrument, including the signalling of attention, feedback, (dis)approval, and dialogue management. In addition, Goodwin (1981) presents compelling evidence for the role of eye gaze in establishing and maintaining participant roles (e.g. securing eye gaze of the primary recipient). These studies paved the way for more recent work that studies eye gaze in more detail, using a variety of methods and linking it to information at the verbal level. In conversation analysis, for instance, gaze behaviour in interaction is studied among others in relation to (dis)engagement, display of

\footnotetext{
${ }^{1}$ Needless to say, the near-exclusive focus on (para)verbal information can at least partly be attributed to technical issues. Early conversation analysis resorted mainly on audio recordings and their transcription, and thus had no direct access to interactionally relevant behavior in the visual modality. Notable exceptions are Goodwin (1981) and Kendon (1967), who made use of video data very early on. With the advent of high-quality and discrete video recording technology, the focus of attention has broadened significantly (see e.g. Mondada 2008).
} 
participation, affiliation, activity types and turn allocation. Drawing on Rossano's (2012a) overview, the following key topics have a long-standing interest in the literature:

- Participation roles:

- Gaze behaviour while speaking differs from gaze while listening, with hearers displaying longer sequences of uninterrupted gaze towards the speaker and speakers constantly shifting their gaze towards and away from the recipient (Kendon 1967, Argyle \& Cook 1976, Goodwin 1981, Vertegaal 1999, Hirvenkari et al. 2013)

- Specific interactional activities require sustained gaze by the recipient, as it is viewed as a display of attention and engagement (e.g. during tellings and questions, Rossano 2012b)

- Unaddressed participants in multi-party interaction seem to anticipate turn shifts between the primary participants. Holler \& Kendrick (2015) show that in questionresponse sequences, the unaddressed participants shift their gaze towards the next speaker before turn completion

- Regulatory functions of gaze

- Speaker gaze can have a 'floor apportionment' function, with speakers displaying gaze aversion briefly after taking the turn, and shifting back towards turn completion in order to signal that the floor may be handed over (Duncan 1975, Kendon 1967, see however De Ruiter 2005 for a somewhat different picture)

- Gaze can be used by speaker to elicit response by the recipients (Goodwin \& Goodwin 1986). This leads to highly synchronized sequences of gaze behaviour across participants, realizing mutual gaze situations (i.e. both participants looking at each other, referred to as gaze windows) in which speakers elicit and recipients realize a form of minimal response (also referred to as back channels or continuers, Bavelas et al. 2002). In case of questions, speaker gaze towards the recipient may serve as an additional resource to communicate that the speaker is expecting a response, especially when the question is not marked intonationally (Rossano 2010)

Although the above-mentioned topics have mainly been developed from a conversation-analytical perspective, other research fields have also developed a growing interest in the role of eye gaze in the interaction. Dialogue models in psycholinguistics and research on human-computer interaction have focused primarily on the role of (mutual) gaze in conversational attention, information uptake and reference resolution. As representative examples of these lines of research Richardson et al. $(2007,2009)$ study the importance of common ground for joint attention by focusing on the coupling of eye movements by speakers and hearers. Gullberg \& Holmqvist (2006) and Gullberg \& Kita (2009) relate addressees' fixations on speakers' gesture to the gesture information uptake. Authors (2015) pursue this line of research and provide evidence for the gestural mimicry as induced by gaze fixations on speakers' gestures: when addressees focus their visual attention on speaker gestures, they are more likely to produce similar gestures in their consecutive turn as speaker after turn transition. Hanna \& Brennan (2007) and Brennan et al. (2008) provide evidence for the role of shared gaze in reference resolution during collaborative tasks. 
In all of these studies, eye gaze is not studied as a single communicative resource that interactants use next to language, but rather as strongly grounded in the interaction and inextricably bound to participant behaviour (be it the participants' gaze behaviour or gesture production).

What unites the studies in the previous paragraph, is their interest in the development of fine-grained methods for studying eye gaze in interaction. One key methodological issue in the study of eye gaze pertains to the reliability of gaze estimations. Although estimations based on video recordings, which is the method that is used in most of the conversation-analytical work, may be useful for a basic segmentation of the distribution of visual attention (e.g. looking at an interlocutor vs. looking away), these estimations are notoriously coarse-grained and unreliable for more detailed analysis (Kendon 2004, Streeck 2009). For instance, video-based analysis does not provide useful information on short fixations on a target (of $200 \mathrm{~ms}$ or less), saccades (i.e. fast movements of the eye) and visual scan paths. In order to be able to include this level of detail in the analysis, a different methodological paradigm is needed, viz. eye-tracking. Eye-tracking or the measuring of gaze points and eye movements during natural behaviour has a long-standing tradition as an instructive paradigm for studying comprehension processes involved in a range of semantic and pragmatic phenomena (e.g. by studying an individual reading, see e.g. Richardson et al. 2007 for an overview). The recently developed unobtrusive eye-tracking systems (e.g. table-top eye-trackers, screen-based eye-trackers or eye-tracking glasses) allow researchers to study eye gaze in naturally occurring interactive settings, in which eye gaze is not only indicative of comprehension processes, but can function as a strong communicative instrument for speakers as well as hearers. This opens up a vast area of research currently being explored in (cognitive) interaction studies, of which the studies listed in the previous paragraph are prime examples.

For the specific topic of turn management in face-to-face interaction, only few studies have applied eyetracking technology to shed a new light on some of the insights from conversation analysis. The abovementioned study by Holler and Kendrik (2015) used eye-tracking glasses in a triadic face-to-face interaction to study visual and verbal cues in turn allocation, and more specifically the gaze behaviour of unaddressed participants as an index of the projection of turn transition. Jokinen et al. (2009) and Jokinen (2010) tracked the gaze behaviour of one participant in 2- and 3-party interactions (using a table-top eyetracker) to study the role of eye gaze in relation to head movements and head gestures as indicative signals in turn-taking. This study suggests that role of eye gaze in turn-taking is less outspoken in triadic than in dyadic interactions, which is attributed to the larger interactional space in which the participants in multiparty conversations need to operate, requiring more visible actions (such as head movements) to attract and retain co-participants' attention. In the present paper, we continue this line of research and use eye-tracking data from two- and three-party face-to-face interactions to explore the correlation and temporal relation between measurable gaze events and specific turn management practices. In the next section, we describe the specific methodological set-up for this study.

\section{Research aim and methodology}


The central goal of this study is to explore how measurable gaze events by speakers and hearers in unscripted face-to-face interaction, collected through the use of mobile eye-tracking, can be linked to specific turn-taking strategies. More specifically, the highly detailed and continuous stream of information provided by the eye-tracker systems allows us to study the sequential-temporal unfolding of gaze events in relation to (a) units in interaction serving specific turn-organizational functions (e.g. an intonation unit with a turn-giving finality), and (b) other verbal or nonverbal elements that serve a function in turn allocation and transition (e.g. filled pauses can be used as a turn-holding strategy). For the purpose of the present paper, we will approach this research question in two steps, corresponding to the two subsections of the analysis section 4 . The first step consists of a general distributional analysis, linking specific gaze events to some of the basic turn management cues described in the literature (4.1.). The second step takes these general distributional data as a starting point to investigate more closely the temporal relationship between relevant gaze events and measurable phenomena the verbal level, such as the onset of a new turn-constructional unit after turn-taking or the onset of a filled pause. By using the timesensitive technique of cross-recurrence analysis, we can gain measure the exact temporal relationship (in terms of co-occurrence or systematic latency) between data points in different time series, as e.g. between discrete units in speech and patterns of nonverbal behaviour such as gesture and gaze (see Coco \& Dale 2014 for an overview).

The data for this paper are taken from the Insight Interaction Corpus (authors 2015), a multimodal corpus of face-to-face interactions in Dutch, transcribed and annotated for gaze and gesture. The corpus consists of conversations between 15 dyads (of about 30 minutes each) and 10 three-party interactions (of about 15 minutes each). The dyadic interactions each consist of three subparts: storytelling, brainstorming and targeted collaborative tasks (on spatial relationships). The three-party interactions are free-range conversations without a predefined topic. In both the two- and three party interactions, the participants were well-acquainted students (age 18-23) and all native speakers of Dutch. For the purpose of this study, we randomly selected 5 dyads in the brainstorming task (which is highly interactive and unscripted) and 4 triads. This selection provides us with a sufficiently large subset to perform the distributional and crossrecurrence analysis, and at the same time may uncover commonalities and differences between two- and three-party interactions.

The screenshots in Figure $1 \mathrm{a}$ and $1 \mathrm{~b}$ show the recording set-up for the two- and three-party interactions. Each figure is a composite of different camera perspectives. In Figure 1a, the image on the left is the perspective of an external camera on the interaction. On the right are the videos from two mobile eyetracking glasses (Arrington Gig-E60), which the interlocutors were wearing during the recording, with the green dots indicating the visual focus of the respective participant. In this specific case, the image on the top right is the output of the scene camera and eye tracker that the girl (on the right in the external perspective) is wearing. The three camera perspectives were edited and synchronised into one video file (from which the figure is a still). In the triad in Figure 1b, the external camera perspective is shown in the bottom right of the image, and this video is synchronized with the output of the three eye-tracking 
systems worn by the participants (Pupil Pro Eye-Tracking Glasses). Again, the eye-tracking system provides both a participant perspective through the scene camera and specific gaze information (red gaze cursor).

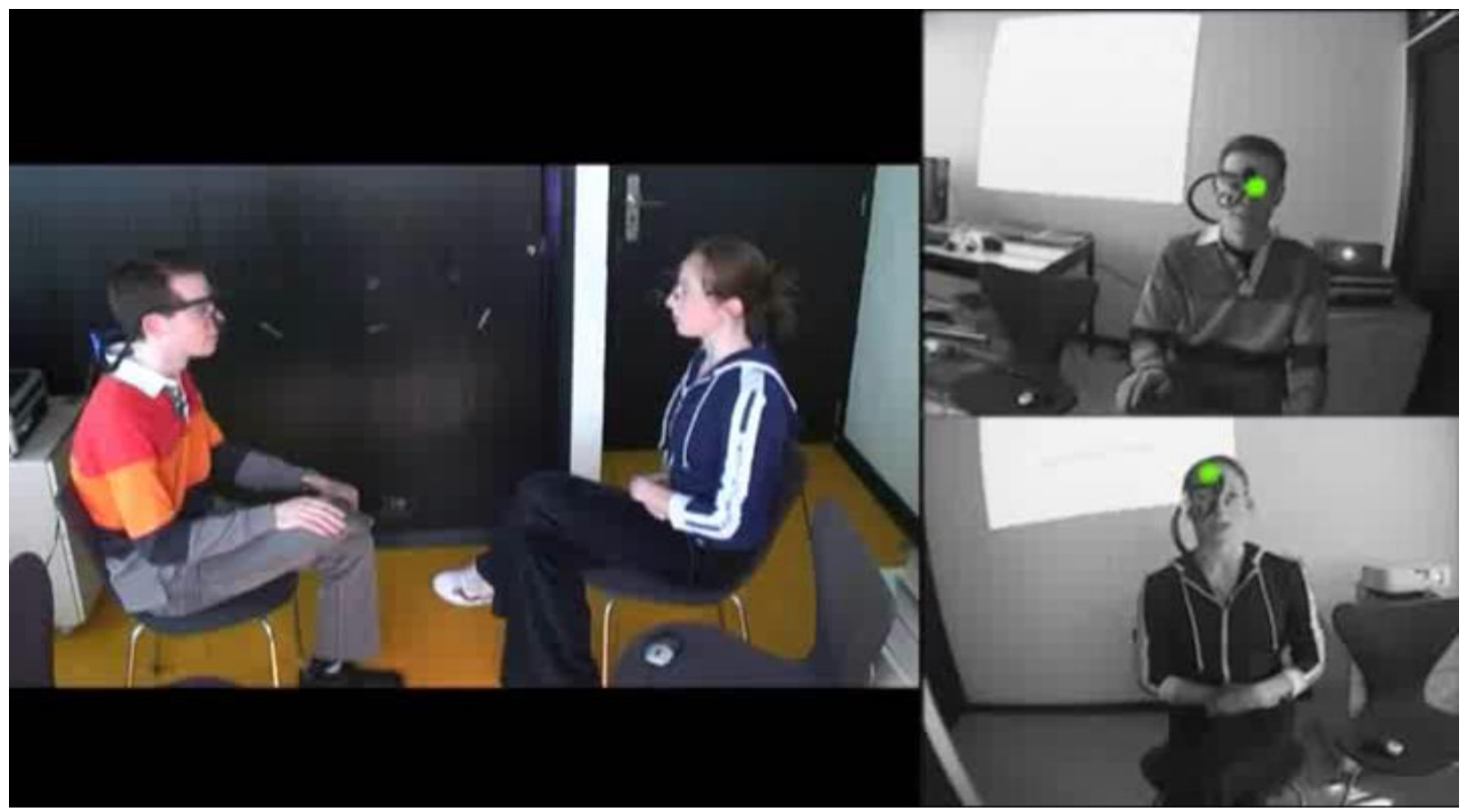

Figure 1a: Recording setup and resulting trivid for the dyadic interactions

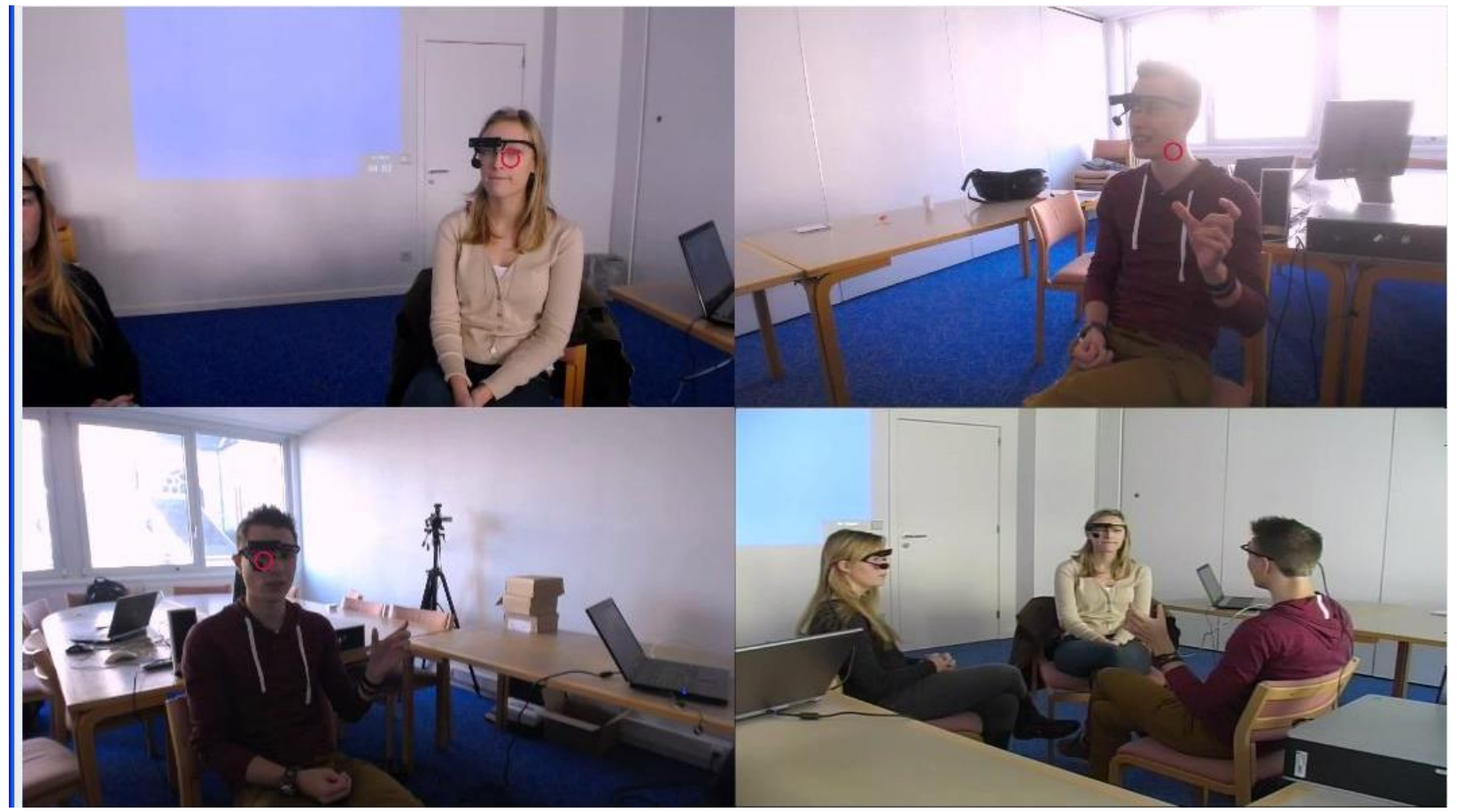

Figure 1b: Recording setup and resulting quadvid for the triadic interactions 
The conversations recorded in the InSight Interaction Corpus were transcribed and annotated in the ELAN multimodal annotation environment (Brugman and Russel 2004; Lausberg and Sloetjes 2009) according to the following principles²:

- The participants' speech is segmented into intonation units, corresponding to segment of speech with a single prosodic contour (Chafe 1994).

- The speech of each participant is represented as a separate tier (or annotation layer) in ELAN and is transcribed according to the modular GAT transcription norm (Selting et al. 1998, Selting 2000). The InSight Interaction Corpus uses an orthographical transcription with additional prosodic information (main accent and terminal intonation contour per intonation unit, pauses within intonation unit, manifest lengthening of vowels and consonants)

- The gaze information for each of the participants is again presented on a different ELAN tier. The continuous information provided by the eye-tracking systems was segmented on the basis of gaze fixations and relevant areas of interest (AOI's). Gaze fixations are moments at which the eyes remain relatively focused on a relevant target (rather than dynamically scanning the scene). These fixations constitute relevant gaze events for the study of gaze behaviour. The minimum fixation duration that allows for a reliable categorization of a gaze event was set at 120 milliseconds. Once the segmentation in discrete gaze fixations is done, these fixations are categorized based on a limited tag set for gaze targets in the interaction: the participant is typically focusing on either the face of one of the co-participants, a gesture of a co-participant, his/her own gesture, or the background. Combining the gaze information of multiple participants simultaneously allows us to calculate moments of mutual gaze, gaze aversion and gaze shifts between participants.

For the purpose of the present study, we additionally annotated the intonation units for turn management features that can be observed. This functional annotation is largely based on the MUMIN coding scheme, which was developed for the study of multimodal human behaviour (Allwood et al. 2007), and which includes the following classical categories:

- Turn take: a speaker takes a turn that wasn't offered

- Turn accept: a speaker accepts a turn that was offered

- Turn hold: the speaker wishes to hold the turn

- Turn yield: the speaker releases the turn under pressure

- Turn elicit: the speaker offers the turn to the interlocutor

- Turn complete: the speaker signals that the turn is about to be concluded and the dialogue can come to an end

Given the fact that we coded these strategies at the level of the intonation unit in this study implies that a single intonation unit can have multiple values for turn management. This is the case, for instance, when a speaker produces a question (which can be categorized as turn elicit), but during the production produces a filled pause in combination with gaze aversion, which can be interpreted as a turn-holding

\footnotetext{
${ }^{2}$ We only discuss the annotation levels that are relevant to the present study. For a full overview of the annotation layers that are provided as part of the InSight Interaction Corpus, including POS-tagging and gesture, see Authors (2015).
} 
strategy. It should be noted, however, that in order to avoid data conflation for specific categories, each intonation unit could only have one value for each of the turn-management strategies. For example, if an intonation unit includes two filled pauses that can be categorized as turn-holding strategies, this was annotated only once. Following this procedure, all the data that were selected for this study were coded independently for turn management features by three independent annotators. The resulting annotations were compared and in those cases where the annotations differed, the sequences were jointly discussed by the three coders until consensus was reached.

Needless to say, this annotation scheme and procedure is relatively course-grained, especially in comparison to the highly detailed descriptions that are typically provided in conversation analysis. We believe, however, that the general classifications at the level of the intonation unit provide a valuable starting point for a corpus-based quantitative approach to multimodal co-occurrences. As a first step, we will focus on the co-occurrence of gaze events with different turn-management strategies. On the basis of the resulting distributions, we will then, in a second step, present in a more fine-grained account of the temporal dynamics of multimodal turn-taking.

\section{Results}

\subsection{Gaze distributions and turn management features}

Table $1 \mathrm{a}$ and $1 \mathrm{~b}$ present the basic distributions for turn management features in relation to gaze behaviour for both the two-party and three-party interactions in the corpus. The pivot tables show all the speakers' intonation units that have a value for each of the turn-management strategies listed in section 3 above, and which exhibit either one of the following gaze patterns:

(i) face (full overlap), meaning that for the entire duration of the intonation unit, the speaker focuses on the face of (one of) the addressees, and

(ii) not face (two-party), referring to cases that involve a moment of gaze aversion between, independent of the question whether this gaze aversion spans of the entire unit, or only occurs at the beginning, the end or in the middle of the intonation unit.

(iii) face shift (only in three-party interactions) indicates that the speaker shifts his/her focus of attention between the two interlocutors at least once in the course of the intonation unit 


\begin{tabular}{|l|l|l|l|}
\hline & Face (full overlap) & Not face & Ratio \\
\hline Turn-H & 130 & 181 & 0,418 \\
\hline Turn-C & 64 & 47 & 0,576 \\
\hline Turn-Y & 38 & 20 & 0,655 \\
\hline Turn-T & 59 & 38 & 0,608 \\
\hline Turn-E & 20 & 8 & 0,714 \\
\hline
\end{tabular}

Table 1a: General distributions for eye gaze and turn-management strategies in dyads

\begin{tabular}{|l|l|l|l|l|}
\hline & Face (full overlap) & Face shift & Not face & Ratio $^{3}$ \\
\hline Turn-H & 29 & 53 & 83 & 0.176 \\
\hline Turn-C & 98 & 75 & 45 & 0.449 \\
\hline Turn-Y & 18 & 21 & 8 & 0.383 \\
\hline Turn-T & 39 & 29 & 12 & 0.487 \\
\hline Turn-E & 89 & 13 & 2 & 0.856 \\
\hline
\end{tabular}

Table 1a: General distributions for eye gaze and turn-management strategies in triads

The absolute frequencies of the occurrences and the ratio scores (calculated as the amount of TCU's with directed at the face over the total amount of TCU's) already point, albeit in a course-grained manner, at a general pattern: intonation units with a turn-holding cue tend to be associated more strongly with moments of gaze aversion and face shift than other dialogue acts. This result, which holds for both the two- and three-party interactions, is very much consistent with the existing literature on the regulatory function of eye gaze in interaction (cf. supra section 2). These basic observations may then serve as the basis for a more fine-grained quantitative and temporal analysis that looks at the occurrence of gaze aversion and gaze shifts at critical turn-holding events, such as transition relevance places, pauses, hesitation markers, etc. This will, in part, be the focus of section 4.2. but as an illustration, we take the sequences in (1) and (2) as prime examples of the general pattern.

In the sequence in (1) the speaker $E$ is reviewing a number of potentially interesting features for a smartphone ${ }^{4}$. At the end of her turn, she considers the possibilities of a little menu, which can be integrated into a new type of cell phone, allowing users to open up a book of recipes, in which one can look for 'different things'. Immediately following this utterance, E. marks her thinking of further options

\footnotetext{
${ }^{3}$ Note that the ratio for the triads was based on the comparison of 'face (full overlap)' and the combination of 'face shift' plus 'not face' as instances of gaze aversion away from a single addressee.

${ }^{4}$ For all the examples discussed in this paper, video clips with all of the interlocutors' perspectives and gaze coordinates will be made available.
} 
by a prolonged verbal filler ' $m h^{\prime}$ '. As far as E's gaze is concerned, on the last word before uttering this filler ('opzoeken', look up), E shifts her gaze from the previously dominant position, which is the interlocutor's face, to the background (marked by [B] in bold face in the gaze tier of line $E(5)$ in example 1), thus averting eye contact with $\mathrm{L}$. This gaze position is maintained throughout the utterance of the verbal filler ' $\mathrm{mhm}$ '. Only at the very end of it, when closing off the articulation of the filler, E re-establishes eye contact with her addressee (marked by [L] in (1)).

(1) Dyad with gaze aversion at transition relevance place

(E\&L 00:33-00:40)

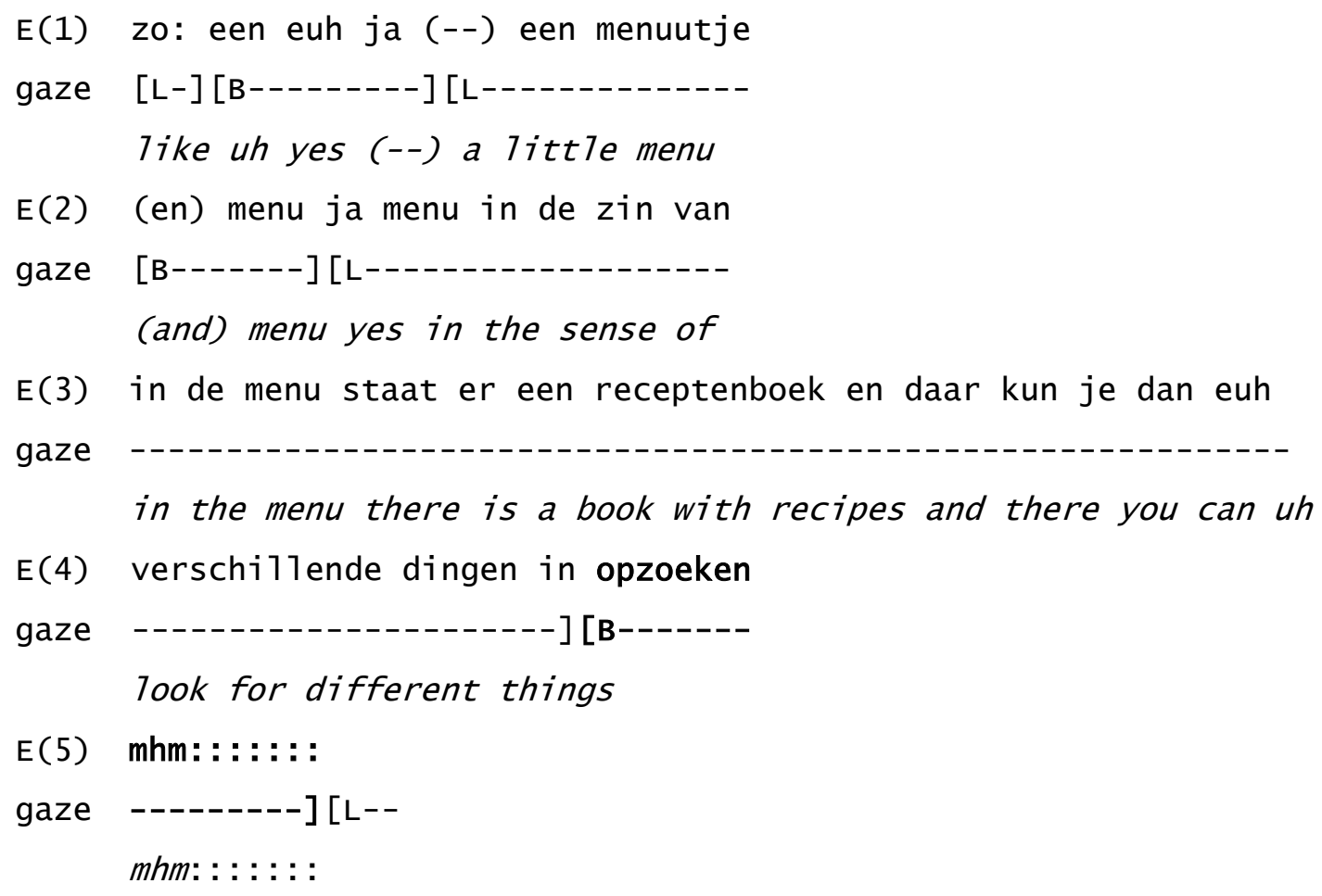

A similar aversion pattern emerges in the triadic sequence in (2), where $X$ shifts his gaze away from his interlocutors' face ( $\mathrm{Y}$ and $\mathrm{Z}$ ) as soon as he starts hesitating when looking for the right word ('student homes'). This slowing down is marked by three speech phenomena, viz. the prolonged articulation of the pronoun 'die' (those), a pause and the prolonged hesitation marker 'euh' (uh). Of particular interest for our current purpose is the observation that this hesitation is marked by an averted eye gaze as well. Immediately after the onset of the pronoun, $X$ 's eye gaze shifts to the background right in the middle between both interlocutors $Y$ and $Z$ (marked by $[B]$ in bold face), where it remains until $X$ resumes his utterance. At the moment he uses the lexical item 'student homes', X's gaze has returned to Z's face, where it remains until almost the end of his utterance. The sequence is closed off by $Y$ acknowledging $X^{\prime} s$ story ('yes'), which draws X's eye gaze towards Y's face. 
(2) triad with gaze aversion at hesitation marker

$$
(X, Y, Z \text { 04:38-04:42) }
$$

X(1) ja kunde of ge hebt zo vaak nog zo van die: (-) euh:

gaze [Y---] [Z-----------------------------] [B----------

yes can you or you have often those (-) uh:

$x(2)$ van die studentenhomes [die] ze dan huren

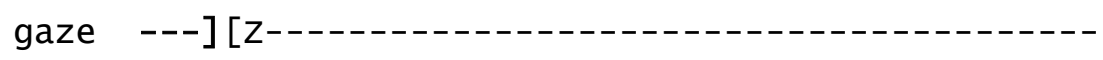

of those student homes, [which] they then rent

Y

[ah ja]

[ah yes]

$x(3)$ [en dan] kunnen wij die van hen huren dan $(-)$ euhm

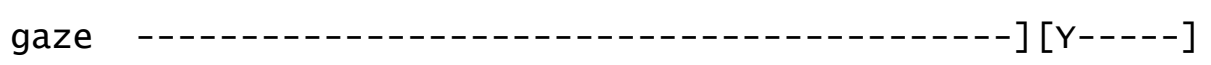

[and then] we can rent those from them then (-) uh:

z [ah ja]

[ah yes]

Y

ja

yes

In addition to the general observation on turn-holding in relation to gaze aversion, a closer look at some of the specific distributional patterns and the underlying TCU's can again be linked to claims made on the basis of qualitative analysis. For instance, we would hypothesize that intonation units with a value 'Turnconclusion' and 'not face' will primarily have gaze fixations away from the addressee(s) at the beginning or in the middle of the unit, and not towards the end. Different authors have argued that speakers tend to look away during a turn and then move back towards the recipient shortly before turn conclusion, either as a function of 'floor appointment' (Kendon 1967, Duncan 1975) or as a multimodal grounding mechanism (Holler \& Bavelas, forthc.), monitoring mutual understanding between the participants. Indeed, if we look at the intonation units with the value Turn- $\mathrm{C}+$ not face in the subcorpus of dyads, 42 of the 47 TCU's have speaker gaze towards the addressee at the end of the turn. The five remaining cases with gaze aversion at turn conclusion all involved the speaker laughing. This observation illustrates that speakers typically establish a strong visual grounding moment at the end of their turn. An example of this prototypical pattern is given in (3), where $L$ wonders what additional functions an innovative cell phone needs to offer. Already during the preceding hesitation marker uttered by R, L's gaze is on the background (B), where it remains even during L's first two TCU's. Only in the third TCU, when the turn is nearing the end on a concrete suggestion ('synchronize with everything'), L creates a visual grounding event by shifting her gaze to her interlocutor $\mathrm{R}$ (marked in bold face). In the case of the three-party interactions, a substantial amount of TCU's involve a moment of gaze shift towards the end of the turn. This again can 
be linked to a central grounding mechanism, with speakers checking for understanding and eliciting feedback on the part of both addressees.

(3) Dyad with re-establishing eye contact at the end of the turn

(R\&L 01:58-02:05)

$\begin{array}{ll}\mathrm{R}: & \text { euhm } \\ \text { gaze } & {[\mathrm{L}-\mathrm{-}]} \\ & \text { uhm }\end{array}$

L(1): Ja wa moet er nog in (.) euh (.) van functies

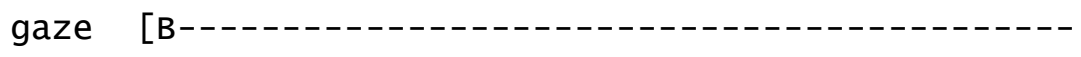

yes what e7se has to be in there (.) uh (.) of functions

L(2): sowieso al ( ) het moet als het vernieuwend moet zijn

gaze

definitely () it must be if it has to be innovative

$L(3)$ : dat het synchroniseert me alles

gaze ----[R---------------------]

that it synchronizes with everything

A turn-management cue that crucially hinges on specific gaze behavior at the onset of TCU is turn-taking, defined in the MUMIN coding scheme as cases in which "the speaker takes a turn that wasn't offered, possibly by interrupting" (Allwood et al. 2007: 9). Taking all intonation units that were coded at Turn-T in the corpus, we would hypothesize that in the instances that have the value 'not face', the moment of gaze aversion or shift does not occur at the onset of the unit. In other words, the turn-taker needs to claim the floor and establish himself or herself as the new speaker, and gazing at interlocutor may be an important strategy in accomplishing this (Rossano 2012a: 315). If we look at the gaze distributions for the 'Turn-T TCU's', this hypothesis seems to be confirmed for both the two-party and three-party conversations. In the dyads, only 2 of the 38 cases with the value 'non-face' involve gaze aversion at the onset of the unit. Even in these two cases, the gaze aversion can be attributed to different factors, as it was already established well before the onset of the relevant unit (and within $200 \mathrm{~ms}$ after the onset of the turn, there is a shift towards the listener's face). In the triads, the new speaker gazes at the previous one at the onset of the turn in 68 out of 80 cases. In 6 instances, the speaker gazes at the third participant in the interaction at the onset of his turn (possible to claim next-speakership). So in both settings, a general gaze pattern or rule (Goodwin 1981:57) seems to emerge that speakers, when taking the turn, should obtain the gaze of the recipient at least at the onset of the turn.

The basic distributional patterns for eye gaze presented thus far, their relationship to specific turnmanagement cues such as turn-holding, turn-conclusion and turn-taking and their relative positioning within TCU's, provide us with a first quantitative insight into the data. In a following step, we will zoom in 
on some of the above-mentioned phenomena in more detail, and will use the full potential of the highly detailed (and continuous) information on participants' gaze behaviour provided by the eye-tracking systems. More specifically, we select two phenomena that illustrate the temporal dynamics of turn-taking:

(1) We investigate the exact timing of gaze events relative to the dialogue act of turn-taking as described above. In other words, what is the typical gaze patterns of turn-takers prior to the onset of new turn (which, as described above, is typically marked by gaze fixations on the previous speaker)

(2) We investigate the strength of multimodal co-occurrences in the realization of specific dialogue acts. Here, we take the case of micro-phenomena in turn exchange (short gaze events, filled pauses) and explore their temporal and functional relationship

\subsection{The temporal dynamics of multimodal turn management}

The highly detailed gaze information generated by (mobile) eye-tracking systems allows us to dig into the synchronicity between gaze behaviour and verbal behaviour. The basic distributions shown in Table 1 above indicated that turn-taking TCU's equally often occur with constant gaze at the conversational partner as with moments of gaze aversion away from the partner. Subsequent analyses (cf. example (3) above) revealed that when there is gaze aversion, the aversion hardly ever ( 8 out of 118 cases) occurs at the beginning of the TCU. In other words, at the actual taking of the turn, i.e. the onset of the turn-taking TCU, there is eye contact between the interlocutors 'battling' to gain the floor. In this section we demonstrate the temporal relation between gaze behaviour of the interlocutor who is (about to be) taking the turn and the onset of the verbal turn-taking TCU itself.

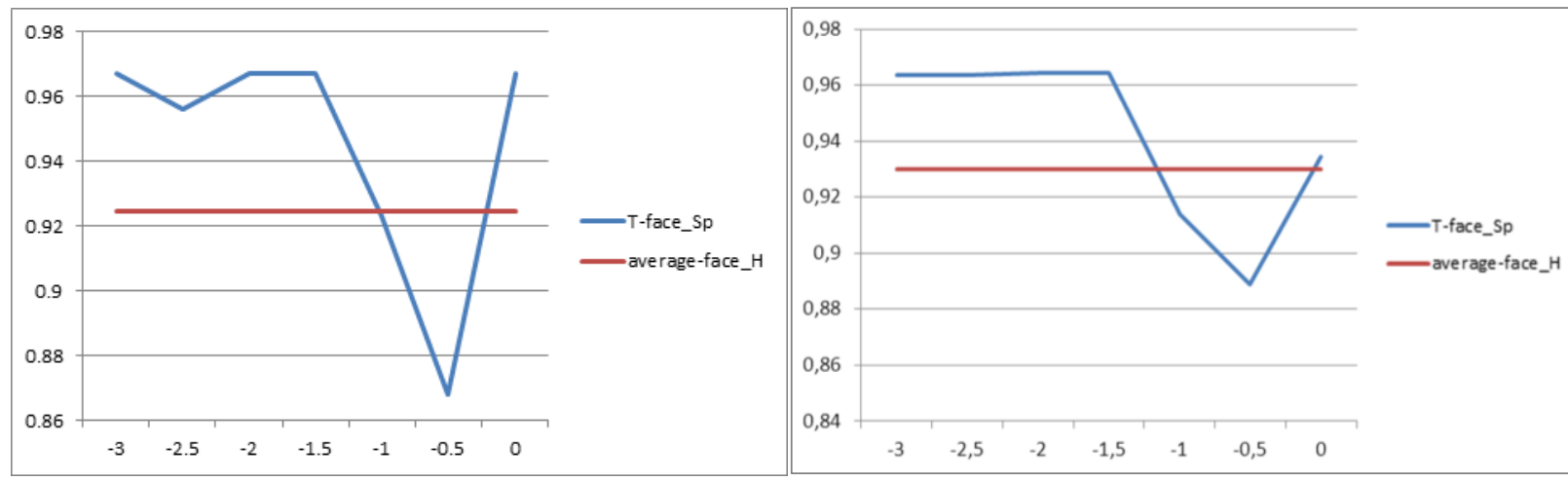

Figure 2a\&b: gaze behaviour prior to the onset of a turn-taking TCU in the two-party (Fig. 2a) and threeparty (Fig. 2b) interactions. The X-axis represents time (prior to the onset of the turn-taking) in seconds; $y$-axis represents the average amount of gaze towards the conversational partner. 
In Figure 2 we see the gaze behaviour of the intervening speaker, i.e. the one taking the turn, up until the onset of the verbal turn-taking TCU itself (value " 0 " on the $x$-axis). The blue line represents the average amount of looking at the conversational partner by the intervening speaker during the 3 seconds prior to the turn-taking intervention (at $\mathrm{T}=0$ on the $\mathrm{x}$-axis). Average values of gaze were calculated per 0.5 second intervals. During the time prior to the actual taking of the turn, the intervening interlocutor is the hearer in the conversation. The red line shows the average amount of time a hearer looks at his speaking partner in our corpus (slightly over 92\%), which is consistent with earlier findings on addressee's gaze behaviour (cf. supra section 2). Plotting both lines (red and blue), we get an indication of what is deviant from default gaze behaviour. Figure 2 indeed demonstrates a high amount of eye-contact at T=0 (over 96\%): intervening speakers typically look at their conversational partner while taking the turn. However, what this graphical representation of the data further demonstrates, is a gaze event prior to the onset of the verbal turn-taking. Typically half a second before this onset (at $\mathrm{T}=-0.5$ on the $\mathrm{x}$-axis), we observe a drop in the amount of eye contact by the intervening speaker. This gaze aversion might be explained as the turntaker's effort to create a gaze event (an observable change in gaze behaviour). Looking at an interlocutor appears not to be a sufficiently strong cue for signalling turn taking: looking at your partner while listening is default (it occurs in roughly $93 \%$ of the time), therefore looking away is what might provide interlocutors with non-verbal ammunition to launch an attack on the conversational floor. The correlation between gaze aversion prior to turn-taking was observed for both dyadic (Figure 2a) and triadic (Figure 2b) conversations.

As demonstrated above, the exact timing of gaze events can play a role in the organization and coordination of turn management. To further explore how gaze events may be temporally anchored to the turn taking machinery, we singled out all cases of gaze aversion with a duration of 500 milliseconds or less. Next, we manually checked with what type of verbal content these nonverbal microphenomena $(n=69)$ coincided. We found that 45 of the gaze aversions occurred while a verbal filler (such as "uhm") was being produced. The use of such fillers has been linked to different functions, including the filler-assymptom in case of production difficulties or planning problems, and the fillers-as-nonlinguistic-signal in conversational floor management (cf. Clark \& Fox Tree 2002 for an overview). In the latter function, fillers have been described as signals that speakers use to keep the floor, very much in line with the literature on gaze aversion as turn-holding signal (cf. supra section 2).

Turning things around, we focussed on the temporal and functional relationship between gaze aversion and these fillers. On a total of 74 hesitation markers in our corpus, 56 (or 76\%) occurred with a brief moment of gaze aversion. To further analyze this synchronisation, we performed cross recurrence analyses in the dyadic (Figure 3a) and triadic (Figure 3b) conversational set-up. A growing body of research (for a recent overview, see Fusaroli et al. 2014) is using this quantification technique of cross recurrence to study phenomena of behaviour matching, including the synchronisation of eye gaze (Richardson \& Dale 2005, Richardson et al. 2009, Dale et al 2011). A cross recurrence analysis is a type of correlation analysis 
that looks for a time lag at which the overlap between two time-series is maximal ${ }^{5}$. In our specific analysis, we want to measure whether gaze aversion and fillers occur at exactly the same time, or whether there might be a systematic time lag between these two events (i.e. the onset of gaze aversion precedes or follows the onset of the hesitation marker). To measure whether and how gaze and verbal behaviour are synchronised in our data, we used the R-package developed by Coco \& Dale (2014) to perform cross recurrence analyses.
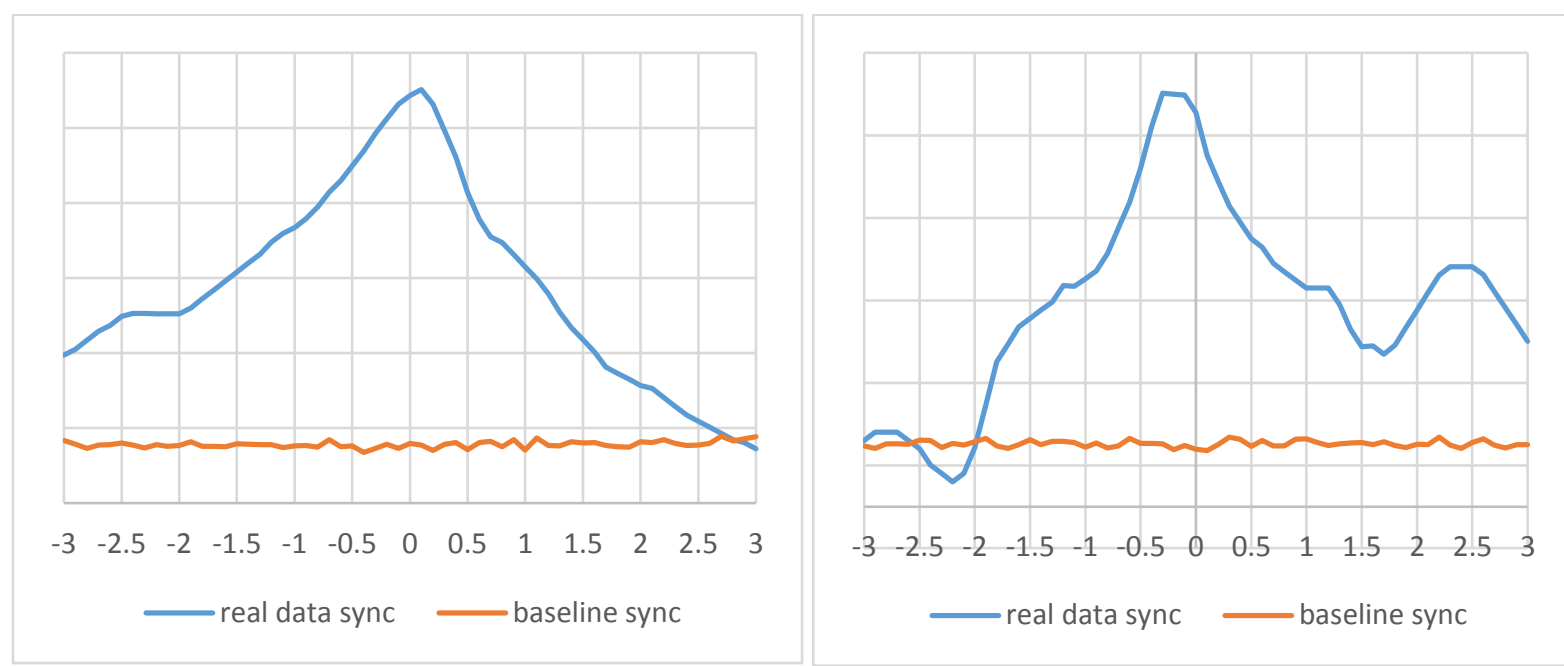

Figure $3 a \& b$ : cross recurrence plots of gaze aversion and hesitation markers for the two-party (Fig. 3a) and three-party (Fig. 3b) interactions

Figures $3 a$ (for dyadic interactions) and $3 \mathrm{~b}$ (for triadic interactions) show a clear bell curve with a peak at or around TO (value " 0 " on the $x$-axis). The presence of the bell curve means there is some kind of synchronisation between the two levels of behaviour under scrutiny (i.c. gaze aversion and fillers): gaze aversions and fillers are not randomly distributed across the conversations, rather, they co-occur with a systematic temporal pattern. The position of the peak of the bell curve indicates whether the temporal relation between the two levels of behaviour is simultaneous (peak at T0), or whether there is a systematic time lag between them. In the case of dyads (Figure 3a) there is near perfect synchronisation, i.e. the onset of gaze aversion and the onset of the filler typically occur at exactly the same time. For the triads (Figure $3 b$ ) there is a very small lag. The peak of the bell curve is just a little ( 200 milliseconds) off to the left of T0. This means that in our triadic conversations, the onset of gaze aversion typically precedes the onset of the filler.

To assess whether this temporal relationship and synchronization between the speech and gaze channels is significant, we applied a random baseline comparison test. This method essentially consists of creating 'fake' conversations by randomly distributing the gaze and speech data (from our actual annotations) over time. With this procedure, in which we follow among others Richardson \& Dale (2005), Louwerse et al.

\footnotetext{
${ }^{5}$ For a detailed explanation of the mathematics behind cross recurrence analyses, see Coco \& Dale (2014). For a more basic introduction to the methodology see Oben (2015).
} 
(2012) and Oben (2015), we created 200 random pairs of speech and gaze data on which we performed a cross recurrence analysis. The average of these 200 analyses can be regarded as the chance level of observing synchronisation between gaze aversion and verbal fillers. The baselines we computed are represented by the red lines in Figure 3. Only if the average of the cross recurrence analyses from the actual data (blue lines in Figure 3 ) are significantly higher than those in the baseline data, we can rule out that the synchronisation we observe is due to chance.

A mixed effects model with conversational dyad or triad as random factor (to rule out that the effect we observed was to a large extent due to one or a few of the dyads/triads) confirms the synchronisation in the actual data is significantly higher than that in the baseline data. This result was obtained both for dyads $(t=5.37, p<0.001)$ and triads $(t=16.64, p<0.001)$. We can therefore conclude that interlocutors tightly, and more often than would be expected by chance, synchronise their hesitation markers with gaze aversion. The tight coupling we observe for this particular phenomenon appeals to the notions of composite signals issued by Clark (1996), multimodal packages raised by McNeill (2006), referring to tight coupling of signals in different semiotic channels.

\section{General discussion}

The present paper was intended as an exploration of how a multimodal approach to interactional language, using state-of-the-art data recording techniques, may contribute to a better understanding of composite signals with specific discourse functions. More specifically, the availability of fine-grained eye gaze data of interlocutors in spontaneous face-to-face interactions, collected with mobile eye-tracking systems, allowed us to study the co-occurrence and synchronization of specific gaze events with verbal or other signals. The results of the general distributional analysis and the case studies with more timesensitive techniques presented in this paper, provide quantitative corpus-based evidence for recurrent multimodal couplings or Gestalts as part of speakers' interactional signal system. Although limited to the analysis of a small set of micro-phenomena, we present this contribution as a more general plea for the inclusion of gaze data (but also other nonverbal resources) in multimodal cognitive-interactional studies that account for the sequential nature of ongoing interactions as sequences of successive usage events (Langacker 2001).

Relating to this sequential dimension, the use of the bidirectional eye-tracking technique in face-to-face interactions provides us with a unique insight into the dynamic and multidimensional process of linguistic interaction. Instead of a 'flat' representation of the speech situation (a bird's eye view perspective), the combination of scene camera data and eye gaze coordinates for all participants in the conversation generates a three-dimensional representation of the interactional landscape, which incorporates the internalized viewpoint of each individual language user as embodied conceptualizer in the interaction. Studies using this methodological framework may contribute to the development of a cognitive linguistic model that maximally embraces the multimodal and multifocal aspects of language use. As the distributional data presented in this paper show, speakers actively engage with the words and eye 
movements of their interlocutors and this again is reflected in the speakers' multimodal behavior itself. This multifocal perspective on interaction presupposes the integration of a dimension of intersubjectivity in the study of linguistic form and meaning (Verhagen 2005): utterances are meaningful not only because they provide a window on the individual's construal of an experience subjective viewpoint, but also because they function as instruments to negotiate construals across different participants.

Finally, the co-occurrence strength and degree of temporal synchronization between elements in different semiotic channels may raise theoretically interesting questions regarding the semiotic boundaries of symbolic units, as traditionally defined among others in Cognitive Linguistics. To take the case presented in this paper: verbal fillers and moments of gaze aversion have both, independently, been described as elements that are conventionally associated with a speaker's turn-holding strategy. The co-occurrence and tight synchronization between these two elements in real-time interaction thus means that we have two symbolic units with a similar function being realized at the same time across modalities. In a recent development of Construction Grammar (CxG), phenomena such as these have been proposed as potential candidates for multimodal constructions (cf. Schoonjans 2014, Schoonjans et al. 2015, Zima 2014, cf. Feyaerts et al. 2016 for an overview). If one considers the basic assumptions of CXG as a usage-based approach, claiming that any recurrent aspect of fully instantiated usage events (be they formal, semantic, pragmatic, verbal or nonverbal) may become part of the conventionalized form-meaning pairing that is the construction, then one should at least theoretically allow for the extension of CxG to multimodal constructions. In this sense, there is no a priori reason to exclude recurrent multimodal co-occurrences involving the tight coupling of specific gaze events with verbal resources as candidates for a multimodal constructicon. Although we cannot discuss the scope and limitations of a multimodal Construction Grammar in the present paper, the results of co-occurrence and recurrence analyses inevitably touch upon the 'frequency of occurrence' or 'recurrent commonalities' criterion that is used to determine construction status in monomodal CxG (e.g. Goldberg 2006).

\section{References}

Allwood, J., et al. 2007. The MUMIN coding scheme for the annotation of feedback, turn management, and sequencing phenomena. International Journal of Language Resources and Evaluation 41, 273287.

Argyle, M. \& Cook, M. 1976. Gaze and Mutual Gaze. London: Cambridge University Press.

Bailly, G., Raidt, S. \& Elisei, F. 2010. Gaze, conversational agents and face-to-face communication. Speech Communication - special issue on Speech and Face-to-Face Communication 52, 598-612.

Baker, Charlotte 1977. Regulators and turn-taking in American Sign Language discourse. In L. A. Friedman (ed.), On the Other Hand: New Perspectives on American Sign Language, 215-236. New York: Academic Press. 
Bavelas J., Coates L. \& Johnson T. 2002. Listener responses as a collaborative process: The role of gaze. Journal of Communication, 566-580.

Beattie, G.W. 1983. Talk: an analysis of speech and non-verbal behaviour in conversation Milton Keynes: Open University Press.

Brennan S., Chen X., Dickinson C., Neider M. \& Zelinsky G. 2008. Coordinating cognition: The costs and benefits of shared gaze during collaborative search. Cognition 106: 1465-1477.

\section{AUTHORS 2015}

Brugman H, Russel A. 2004. Annotating Multimedia/Multi-modal resources with ELAN. In: Proceedings of the Fourth International Conference on Language Resources and Evaluation (LREC).

Chafe, W. 1994. Discourse, Consciousness, and Time: The Flow and Displacement of Conscious Experience in Speaking and Writing. Chicago: University of Chicago Press.

Cienki, A. \& Müller, C. (eds.) 2008. Metaphor and Gesture. Amsterdam/Philadelphia: John Benjamins.

Clark, H.H. 1996. Using Language, Cambridge: Cambridge University Press.

Clark, H.H. \& Fox Tree, J.E. 2002. Using uh and um in spontaneous speaking. Cognition 84, 73-111.

Coco, M. I. \& Dale, R. 2014. Cross-recurrence quantification analysis of categorical and continuous time series: an R package. Frontiers in Quantitative Psychology and Measurement 5, 1-14.

Dale, R., Warlaumont, A. S. \& Richardson, D. C. (2011). Nominal cross recurrence as a generalized lag sequential analysis for behavioral streams.International Journal of Bifurcation and Chaos, 21, 11531161.

Dancygier, B. \& Sweetser, E. (eds.) 2015. Viewpoint in Language: A Multimodal Perspective. Cambridge: Cambridge University Press.

Deppermann, A. 2013a. Introduction: Multimodal interaction from a conversation analytic perspective." Journal of Pragmatics 46-1, 1-7.

Deppermann, A. 2013b. Turn-design at turn-beginnings: Multimodal resources to deal with tasks of turnconstruction in German." Journal of Pragmatics 46, 1, pp. 91-121

De Ruiter, J. P. 2005. The role of eye-gaze in visual dialogue tasks. Paper presented at the AMLaP, Ghent, Belgium.

Drew, P. \& Heritage, J. (eds.) 2006. Conversation Analysis. London: Sage.

Duncan , S. 1975. Interaction units during speaking turns in dyadic, face - to - face conversations. In: A. Kendon, R. M. Harris \& M. R. Key (Eds.), Organization of Behavior in Face-to-Face Interaction. The Hague: Mouton 199-212.

Emmorey, K. Thompson, R. \& Colvin, R. 2009. Eye Gaze During Comprehension of American Sign Language by Native and Beginning Signers. Journal of Deaf Studies and Deaf Education 14:2, 237-243.

Authors 2016

Fusaroli, R., Konvalinka, I. \& Wallot, S. 2014. Analyzing Social Interactions: The Promises and Challenges of Cross Recurrence Quantification Analysis, Springer Proceedings for Mathematics.

Goldberg, A.E. 2006. Constructions at Work. The nature of generalization in language. Oxford: Oxford University Press.

Goodwin, C. 1980. Restarts, pauses, and the achievement of a state of mutual gaze. Sociological Inquiry 272-302. 
Goodwin, C. 1981. Conversational Organization. Interaction between Speakers and Hearers. New York, London.

Goodwin, M. H., \& Goodwin, C. 1986. Gesture and coparticipation in the activity of searching for a Word. Semiotica, 62(1/2), 51-75.

Gullberg M. \& Holmqvist, K. 2006. What speakers do and what addresses look at: Visual attention to gestures in human interaction live and on video. Pragmatics and Cognition 14:1, 53-82.

Gullberg M. \& Kita S. 2009. Attention to speech-accompanying gestures: Eye movements and information uptake. Journal of Nonverbal Behaviour 33, 251-277.

Hanna J. \& Brennan S. 2007. Speakers' eye gaze disambiguates referring expressions early during faceto-face conversation. Journal of Memory and Language 57: 596-615.

Hirvenkari L., Ruusuvuori J, Saarinen V-M, Kivioja M, Peräkylä A, et al. 2013. Influence of Turn-Taking in a Two-Person Conversation on the Gaze of a Viewer. Finland. PLoS ONE 8(8): e71569. doi:10.1371/journal.pone.0071569.

Holler, J. \& Kendrick, K. 2015. Unaddressed participants' gaze in multi-person interaction: Optimizing recipiency. Frontiers in Psychology, 6: 98.

Holler, J., \& Bavelas, J.B. Multi-Modal Communication on Common Ground. A Review of Social Functions. Accepted for R. B. Church, M. W. Alibali, \& S. Kelly (Eds.), Why gesture? How the hands function in speaking, thinking, and communicating. Amsterdam NL: Benjamins.

Jokinen, K. 2010. Non-verbal signals for turn-taking \& feedback. Proc. of 7th Int. Conf. on Language Resources \& Evaluation.

Jokinen, K. Nishida, M. \& Yamamoto, S. 2009. Eye gaze experiments for conversation monitoring. In: Proceedings of the 3rd International Universal Communication Symposium.

Kawase, S. 2014a. Assignment of Leadership Role Changes Performers' Gaze During Piano Duo Performances. Ecological Psychology 26:3, 198-215.

Kawase, S. 2014b. Gazing behavior and coordination during piano duo performance. Attention, Perception, \& Psychoph., 76, 527-540.

Kendon, A. 1967. Some functions of gaze-direction in social interaction. Acta Psychologica 26, 22-63.

Kendon, A. 2004. Gesture: Visible Action as Utterance. Cambridge: Cambridge University Press.

King, E., \& Ginsborg, J. 2011. Gestures and glances: interactions in ensemble rehearsal. New Perspectives of Music and Gesture. Surrey: Ashgate, 177-202.

Langacker, R.W. 2001. Discourse in Cognitive Grammar. Cognitive Linguistics 12-2, 143-188.

Lausberg H, Sloetjes H. 2009. Coding gestural behavior with the NEUROGES-ELAN system. Behavior Research Methods, Instruments, \& Computers 41: 841-849

Linell, P. 2009. Rethinking language, mind, and world dialogically. Charlotte, NC: Information Age Publishing.

McNeill, D. 2006. Gesture, gaze, and ground. In: S. Renals \& S. Bengio (Eds.): Proceedings of Machine learning for multimodal interaction: Second international workshop 2005, Berlin/Heidelberg: Springer Verlag, 1-14. 
McNeill, D. 2006. Gesture, gaze, and ground. In: S. Renals \& S. Bengio (Eds.): Proceedings of Machine learning for multimodal interaction: Second international workshop 2005, Berlin/Heidelberg: Springer Verlag, 1-14.

Mondada, L. 2007. Multimodal resources for turn-taking: pointing and emergence of next speakers. Disc. Studies 194-225.

Mondada, L. 2008. Using Video for a Sequential and Multimodal Analysis of Social Interaction: Videotaping Institutional Telephone Calls". Forum Qualitative Sozialforschung / Forum: Qualitative Social Research, vol. 9, no. 3.

Mondada, L. 2013. Embodied and spatial resources for turn-taking in institutional multi-party interactions: Participatory democracy debates. Journal of Pragmatics, 46-1, pp. 39-68

Müller et al. (eds.) 2014. Body - Language - Communication: An International Handbook on Multimodality in Human Interaction. Berlin: Mouton De Gruyter.

AUTHORS 2015

AUTHORS 2015

Pickering M. \& Garrod S. 2004. Toward a mechanistic psychology of dialogue. Behavioral and Brain Sciences 27: 169-226.

Pinar Sanz, M. (ed.) 2013. Multmodality and Cognitive Linguistics. Amsterdam/Philadelphia: John Benjamins.

Quek, F., R.T. Rose \& D. McNeill. 2005. Multimodal meeting analysis. International Conference on Intelligence Analysis.

Richardson, D. \& Dale, R. 2005. Looking to understand: The coupling between speakers' and listeners' eye movements and its relationship to discourse comprehension. Cognitive Science 29, 1045-1060.

Richardson, D.C., Dale, R. \& Spivey, M. 2007. Eye movements in language and cognition. A brief introduction. In: M. Gonzalez-Marquez, I. Mittelberg, S. Coulson \& M. Spivey (eds.), Methods in Cognitive Linguistics. Amsterdam/Philadelphia: John

Richardson, D., et al. 2009. Conversation, gaze coordination \& beliefs about context. Cogn. Science 14681482.

Rossano, F. 2010. Questioning and responding in Italian. Journal of Pragmatics, 42(10), 2756-2771.

Rossano, F. 2012a. Gaze in conversation. In: J. Sidnell \& T. Stivers (eds.), The Handbook of Conversation Analysis. Chichester: Wiley-Blackwell, 308-329.

Rossano, F. 2012b. Gaze behavior in face - to - face interaction . Unpublished PhD Dissertation, Max Planck Institute for Psycholinguistics, Nijmegen, The Netherlands.

Sacks, H., E.A. Schegloff \& G. Jefferson. 1974. A simplest systematics for the organisation of turn-taking for conversation. Language 50, 696-735.

Schegloff, E.A. 2007. Sequence Organization in Interaction. Cambridge: Cambridge University Press.

Schegloff, E. A. 1982. Discourse as an interactional achievement: some uses of "uh huh" and other things that come between sentences. In D. Tannen (Ed.), Analyzing discourse: text and talk. Georgetown University Roundtable on Languages and Linguistics 1981 (pp. 71-93). Washington, DC: Georgetown University Press. 
Schoonjans, Steven. 2014a. Modalpartikeln als multimodale Konstruktionen. Eine korpusbasierte Kookkurrenzanalyse von Modalpartikeln und Gestik im Deutschen. Unpublished PhD thesis, University of Leuven.

Schoonjans, Steven, Geert Brône, and Kurt Feyaerts. 2015. Multimodalität in der Konstruktionsgrammatik: Eine kritische Betrachtung illustriert anhand einer Gestikanalyse der Partikel einfach. In Jörg Bücker, Susanne Günthner and Wolfgang Imo (eds.), Konstruktionsgrammatik V. Konstruktionen im Spannungsfeld von sequenziellen Mustern, kommunikativen Gattungen und Textsorten, Tübingen: Stauffenburg, 291-308.

Schmitt, R. 2005. Zur multimodalen Struktur von turn-taking, Gesprächsforschung 6, 17-61.

Selting, M., Auer P, Barden B, Couper-Kuhlen E, Günther S, Quasthoff U, Schlobinski P, Uhmann S. 1998. Gesprachsanalytisches Transkriptionssystem (GAT). Linguistische Berichte 173: 91-122.

Selting, M. 2000. The construction of units in conversational talk. Language in Society 29: 477-517. Sidnell, J. \& Stivers, T. (eds.) 2013. The Handbook of Conversation Analysis. London: Wiley-Blackwell. Streeck J. 2009. Gesturecraft - The Manufacture of Meaning. Amsterdam/Philadelphia: John Benjamins. Verhagen, A. 2005. Constructions of Intersubjectivity. Oxford: Oxford University Press.

Verhagen, A. 2008. Intersubjectivity and the architecture of the language system. In: Jordan Zlatev, Timothy P. Racine, Chris Sinha, Esa Itkonen (eds.), The Shared Mind: Perspectives on Intersubjectivity. Amsterdam/Philadelphia: John Benjamins Publishing Company, 307-331.

Vertegaal, R. 1999. The GAZE Groupware system: Mediating Joint Attention in Multiparty Communication and Collaboration. In: Proceedings of CHI'99. Pittsburgh, ACM Press, 294-301.

Vertegaal, R., Slagter, R., Van der Veer, G. \& Nijholt, A. 2001. Eye gaze patterns in conversations: There is more to conversational agents than meets the eyes. In: Proceedings of the Conference on Human Factors in Computing Systems.

Zima, Elisabeth. 2014. Gibt es multimodale Konstruktionen? Eine Studie zu [V(motion) in circles] und [all the way from X PREP Y]. Gesprächsforschung - Online-Zeitschrift zur verbalen Interaktion 15, 1-48. 